\title{
MicroRNA and mRNA profiling in the idiopathic inflammatory myopathies
}

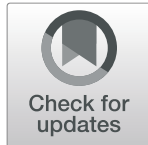

Joanna E. Parkes ${ }^{1,2^{*}}$, Anastasia Thoma ${ }^{3}$, Adam P. Lightfoot ${ }^{3}$, Philip J. Day ${ }^{4,5}$, Hector Chinoy $^{6,7,8}$ and Janine A. Lamb ${ }^{1}$

\begin{abstract}
Background: The idiopathic inflammatory myopathies (IIMs) are heterogeneous autoimmune conditions of skeletal muscle inflammation and weakness. MicroRNAs (miRNAs) are short, non-coding RNA which regulate gene expression of target mRNAs. The aim of this study was to profile miRNA and mRNA in IIM and identify miRNAmRNA relationships which may be relevant to disease.

Methods: mRNA and miRNA in whole blood samples from 7 polymyositis (PM), 7 dermatomyositis (DM), 5 inclusion body myositis and 5 non-myositis controls was profiled using next generation RNA sequencing. Gene ontology and pathway analyses were performed using GOseq and Ingenuity Pathway Analysis. Dysregulation of miRNAs and opposite dysregulation of predicted target mRNAs in IIM subgroups was validated using RTqPCR and investigated by transfecting human skeletal muscle cells with miRNA mimic.

Results: Analysis of differentially expressed genes showed that interferon signalling, and anti-viral response pathways were upregulated in PM and DM compared to controls. An anti-Jol autoantibody positive subset of PM and DM $(n=5)$ had more significant upregulation and predicted activation of interferon signalling and highlighted T-helper (Th1 and Th2) cell pathways. In miRNA profiling miR-96-5p was significantly upregulated in PM, DM and the anti-Jo1 positive subset. RTqPCR replicated miR-96-5p upregulation and predicted mRNA target (ADK, CD28 and SLC4A10) downregulation. Transfection of a human skeletal muscle cell line with miR-96-5p mimic resulted in significant downregulation of ADK.
\end{abstract}

Conclusion: MiRNA and mRNA profiling identified dysregulation of interferon signalling, anti-viral response and Thelper cell pathways, and indicates a possible role for miR-96-5p regulation of ADK in pathogenesis of IIM.

Keywords: Idiopathic inflammatory myopathies, Polymyositis, Dermatomyositis, microRNA, RNA sequencing

\section{Background}

The idiopathic inflammatory myopathies (IIMs) are a heterogeneous group of autoimmune conditions characterised by weakness and inflammation of skeletal muscle. Major clinical classifications of IIM include polymyositis (PM), dermatomyositis (DM) and inclusion body

\footnotetext{
* Correspondence: jparkes@binghamton.edu

${ }^{1}$ Centre for Epidemiology, Division of Population Health, Health Services Research \& Primary Care, Faculty of Biology, Medicine and Health,

Manchester Academic Health Science Centre, University of Manchester, Manchester, UK

${ }^{2}$ Stopford Building, University of Manchester, Oxford Road M13 9PT, Manchester, UK

Full list of author information is available at the end of the article
}

myositis (IBM). Between 60 and $70 \%$ of IIM patients have detectable myositis associated or specific autoantibodies which are associated with particular clinical phenotypes [1].

MicroRNAs (miRNAs) are short, non-coding, singlestranded RNAs which target particular mRNAs for translational suppression and/or degradation. In this manner miRNAs 'fine-tune' gene expression and influence a wide variety of cellular processes and pathways. MiRNAs which regulate the immune system have been found to be dysregulated in autoimmune conditions including IIM [2] and miRNAs important in the development and maintenance of skeletal muscles, termed

C The Author(s). 2020 Open Access This article is licensed under a Creative Commons Attribution 4.0 International License, which permits use, sharing, adaptation, distribution and reproduction in any medium or format, as long as you give appropriate credit to the original author(s) and the source, provide a link to the Creative Commons licence, and indicate if changes were made. The images or other third party material in this article are included in the article's Creative Commons licence, unless indicated otherwise in a credit line to the material. If material is not included in the article's Creative Commons licence and your intended use is not permitted by statutory regulation or exceeds the permitted use, you will need to obtain permission directly from the copyright holder. To view a copy of this licence, visit http://creativecommons.org/licenses/by/4.0/. The Creative Commons Public Domain Dedication waiver (http://creativecommons.org/publicdomain/zero/1.0/) applies to the data made available in this article, unless otherwise stated in a credit line to the data. 
myomiRs, have been found to be dysregulated in IIM [3, 4]. MiRNA dysregulation has been investigated in IIM subtypes in a variety of tissues but further research is required to understand the role of microRNA in IIM pathology [5].

In this study we used next generation RNA sequencing to generate a comprehensive miRNA and mRNA profile in whole blood of PM, DM and IBM patients and then followed up particular miRNA-mRNA interactions by transfecting a human skeletal muscle cell line with miRNA mimic.

\section{Methods}

\section{Study population}

Patients with definite IIM were recruited as part of the Salford Royal NHS Foundation Trust myositis research tissue bank (MRTB). All patients fulfilled Bohan and Peter criteria for PM or DM $[6,7]$ and Griggs or European Neuromuscular Centre or Medical Research Council criteria for IBM $[8,9]$. Control samples were age and ethnicity matched from MRTB taken for diagnostic or treatment purposes (non-myositis controls). All clinical data was held on the EuroMyositis database [10]. Demographic features for the 7 PM, 7 DM, 5 IBM patients and 5 non-myositis controls included in this study are summarised in Table 1. Disease duration ranged from $<1$ year to 10 years at time of blood sample. On average, disease duration was slightly higher in the IBM group (4.2 years) compared to PM, DM or anti-Jo1 positive groups $(<3.2,<1.7$ and $<3.4$ years respectively). One antibody negative PM patient had been diagnosed with cancer at the time of blood draw (in situ intraepithelial non-infiltrating carcinoma).

\section{RNA preparation and sequencing}

Total RNA was extracted from whole blood using Mag$\mathrm{MAX}^{\mathrm{sm}}$ for stabilised blood tubes RNA Isolation Kit (Ambion). RNA concentration and integrity was measured using Agilent RNA 6000 Nano chips on the Bioanalyzer 2100. Complementary DNA (cDNA) library preparation was performed using NEBNext ${ }^{\circ}$ Multiplex Small RNA Library Prep sets for Illumina using 6\% PolyAcrylamide gel to perform size selection for miRNA cDNA libraries. Illumina TruSeq ${ }^{\circ}$ Stranded mRNA library preparation kit was used for mRNA. Paired end sequencing was performed for both libraries on an Illumina HiSeq 4000. Samples were run 8 to a lane with a read depth of approximately 39 million per sample.

\section{RNA sequencing analysis}

FastQC was used to produce quality reports for each sample (read 1 and read 2, refer to Additional file 1 for details of quality control methods). Reads were trimmed using Trimmomatic, mapped to the human genome (hg38, gencode v25) using STAR, counted using htseq, and differential gene expression was analysed using DESeq2.

\section{Gene ontology and pathway analyses}

The R package 'GOseq' was used to analyse significantly differentially expressed (DE) genes and identify over or under-represented gene ontology terms (see Additional file 1 for details).

QIAGEN's Ingenuity Pathway Analysis (IPA) (IPA', QIAGEN Redwood City, www.qiagen.com/ingenuity) 'Canonical Pathways' tool was used to identify pathways with an enrichment of significantly DE genes and the 'MicroRNA Target Filter' tool was used to match significantly DE miRNA $(p<0.01)$ to predicted or experimentally observed mRNA targets with significant $(F D R<$ 0.05) opposite expression changes in the mRNA RNA sequencing data (see Additional file 1 for details).

\section{RTqPCR}

cDNA synthesis was performed using the high capacity RNA-to-cDNA ${ }^{\mathrm{Tx}}$ kit for mRNA and the TaqMan ${ }^{\circ}$ Advanced miRNA cDNA Synthesis Kit for miRNA (Applied Biosystems). RTqPCR was performed on the QuantStudio $^{\mathrm{m}} 12 \mathrm{k}$ Flex (Applied BioSystems) using TaqMan ${ }^{\circ}$ Gene Expression and Advanced miRNA Assays (full list of assays in Additional Table 1). Relative expression was calculated using the $2^{-\Delta \Delta \mathrm{Ct}}$ method compared to reference genes (PRDM4 and UBE2D2 for mRNA, miR-503$5 \mathrm{p}$ and miR-425-5p for miRNA assays).

\section{Transfection of human skeletal muscle cell line}

An immortalised human skeletal muscle cell line, generated from primary human myoblasts, was cultured [11]

Table 1 Demographic and autoantibody status information for samples

\begin{tabular}{lllll}
\hline & PM $(n=7)$ & DM $(n=7)$ & IBM $(n=5)$ & Control $(n=5)$ \\
\hline \% Female & 57.1 & 57.1 & 40 & 20 \\
Mean age & $59.3 \pm 7.2$ & $50.6 \pm 14.4$ & $65.2 \pm 4.6$ & $44.6 \pm 10.7$ \\
Myositis Specific or Associated Autoantibodies & 4 anti-Jo1 & 1 anti-Jo1 & Negative & Negative \\
& & 1 anti-MDA5 & & \\
& & 1 anti-SAE & & \\
\hline
\end{tabular}


(refer to Additional file 1 for details). Cells were seeded at 40,000 cells per well and incubated at $37^{\circ} \mathrm{C}$ for $24 \mathrm{~h}$ before transfection. Transfection was performed using Lipofectamine RNAiMAX and mirVana ${ }^{\text {ta }}$ miRNA mimic for hsa-miR-96-5p (Assay ID MC10422) or negative control (Negative Control \#1, Ambion). After transfection, cells were incubated at $37^{\circ} \mathrm{C}$ for $24 \mathrm{~h}$. Total RNA was isolated from cells using TRIzol $^{\circ}$ Reagent (Invitrogen) and gene expression measured by RTqPCR as described above.

\section{Statistical analyses}

RNAseq and pathway analyses false discovery rates were calculated using the Benjamini-Hochberg method.

$P$-values for the RTqPCR analyses were calculated using an independent $\mathrm{T}$-test on delta $\mathrm{Ct}$ values from each sample (Experimental Ct-Reference $\mathrm{Ct}$ ) in the subgroups.

\section{Results}

Differentially expressed genes and microRNAs in IIM patients compared to controls

Overall, 129, 53 and 24 DE mRNAs were identified for PM, DM and IBM compared to controls, respectively (false discovery rate $(\mathrm{FDR})<0.05)$ (Fig. 1a, Additional file 2). Analysis of the five anti-Jo-1 positive samples (4 PM and $1 \mathrm{DM}$ ) compared to controls identified $691 \mathrm{DE}$ genes (Fig. 1a, Additional file 2). In analysis of miRNAs, 4, 4, 7 and 3 miRNAs were DE for the PM, DM, IBM and anti-Jo-1 positive subgroups compared to controls, respectively $(p<0.01)$ (Fig. 1b, Additional file 3$)$. No DE miRNAs were identified at FDR $<0.05$.
Innate immunity pathways are enriched for dysregulation in PM and DM and the anti-Jo1 positive subset

GOseq analysis of genes differentially expressed in the PM, DM and anti-Jo1 subgroups compared to controls found that the categories with the most significant overrepresentation of DE genes (Benjamini Hochberg adjusted $p$-value $<0.05)$ were immune-related responses including responses to type I interferon and viruses (Additional file 4).

Similarly, when IPA was used to identify canonical pathways with an over-representation of DE genes 'Interferon signalling' was the most significantly dysregulated canonical pathway for the PM, DM and anti-Jo1 subgroups and predicted to be upregulated in all three groups (- $\log p$-value $>1.3$ significant for pathway dysregulation and a z-score $>2$ or $<-2$ significant for predicted activation or inhibition, respectively) (Fig. 2, Additional file 5). The most significant pathways for PM were interferon or interleukin (IL-6, IL-15 and IL-7) related and highlighted roles for JAK kinases in the signalling of both of these groups of cytokines. The five most significant pathways for DM were related to interferon signalling and anti-viral innate immunity; particularly the role of pattern recognition receptors, interferon regulatory factors and STAT3 (Additional file 5). Grouping by anti-Jo1 positivity similarly identified pathways related to interferon, interleukin, JAK kinases and antiviral innate immunity. Notably, the anti-Jo1 subgroup had a higher ratio of dysregulated molecules to total molecules in the 'Interferon signalling' pathway, a higher proportion of molecules exclusive to type II interferon signalling, including upregulation of Interferon gamma receptor 1, and a higher activation score (z-score) compared to the other clinical groups. Additionally, the anti-
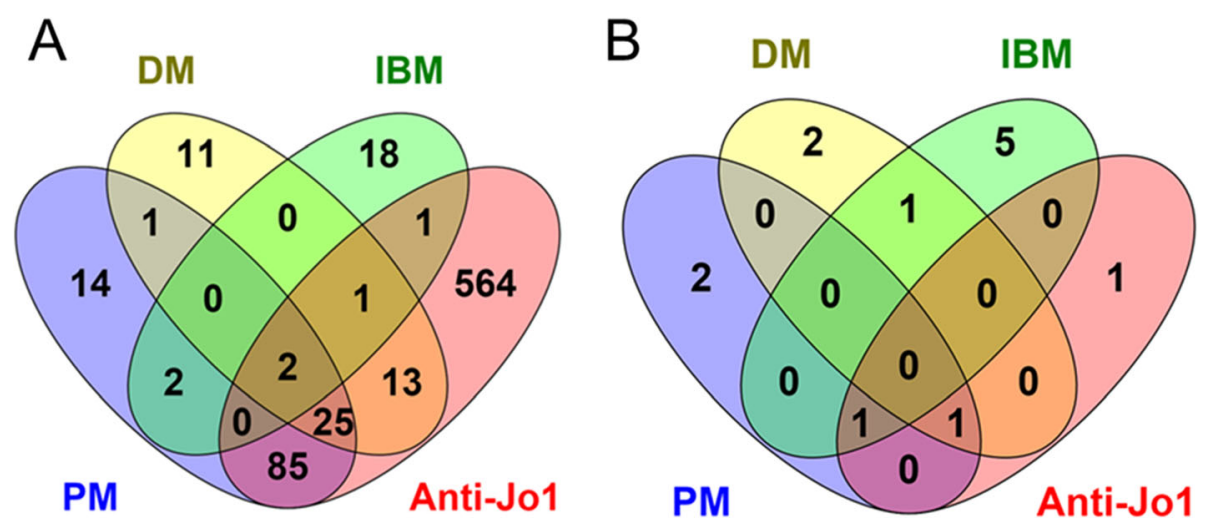

Fig. 1 Significantly differentially expressed mRNA (a) and microRNA (b) in idiopathic inflammatory myopathy subgroups versus controls DM Dermatomyositis, PM Polymyositis, IBM Inclusion Body Myositis Anti-Jo1 Anti-Jo1 autoantibody positive samples Generated from lists of significantly differentially expressed mRNA (FDR < 0.05) and microRNA ( $p<0.01)$ in RNA sequencing of whole blood from 7 PM, 7 DM, 5 IBM and 5 controls (4 PM and 1 DM were anti-Jo1 autoantibody positive) using Venny 2.1 [Oliveros, J.C. (2007-2015) Venny. An interactive tool for comparing lists with Venn's diagrams. http://bioinfogp.cnb.csic.es/tools/venny/index.html] 


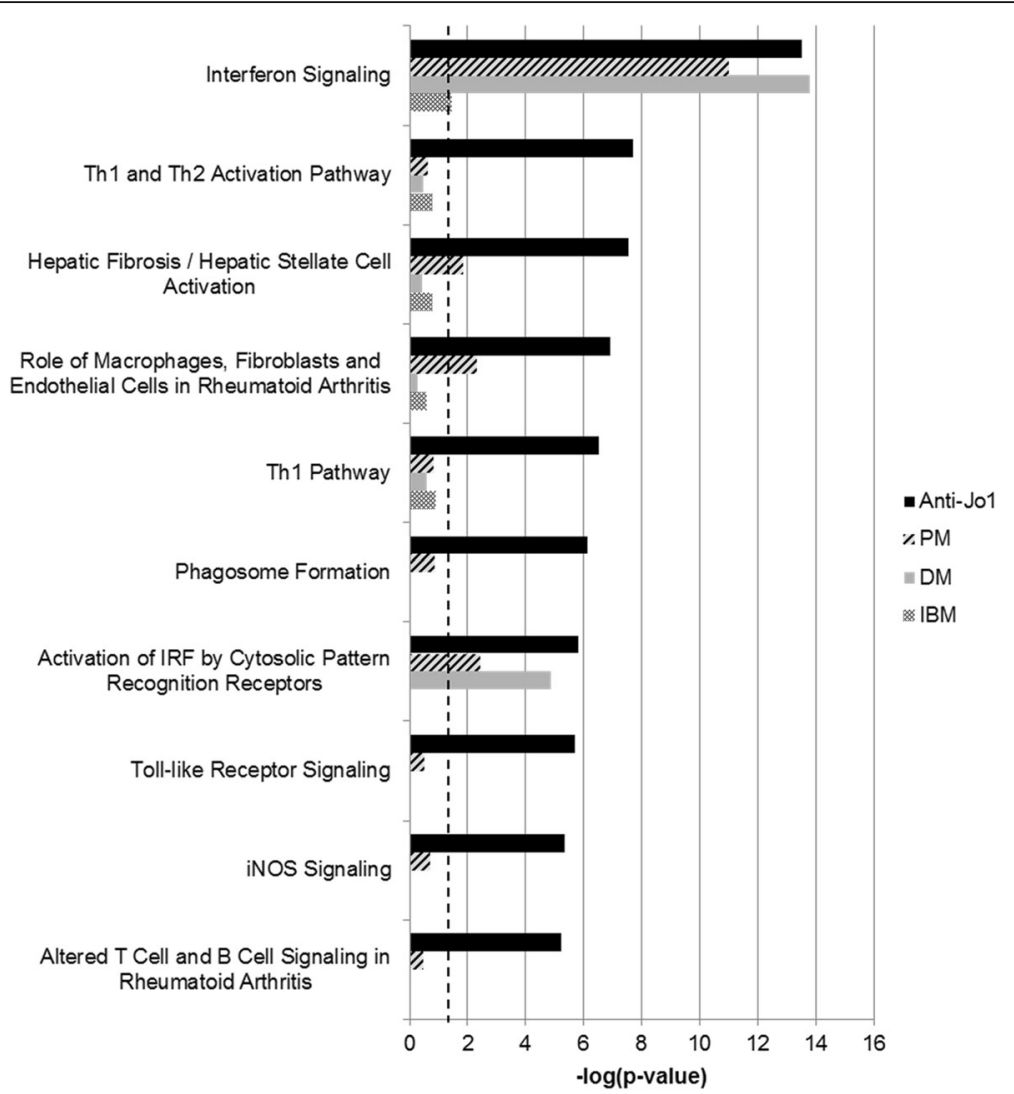

Fig. 2 Top ten canonical pathways enriched for dysregulation in anti-Jo1 positive samples compared to controls PM Polymyositis, DM Dermatomyositis, IBM Inclusion Body Myositis, Anti-Jol Anti-Jo1 autoantibody positive subset of PM and DM, Th T-helper cell, IRF Interferon regulatory factor, NF-KB, nuclear factor kappa-light-chain-enhancer of activated B cells, iNOS Inducible nitric oxide synthase. Data generated using QIAGEN's Ingenuity Pathway Analysis 'Canonical Pathways' tool on differential expression data from RNA sequencing of whole blood from 7 PM, 7 DM, 5 IBM and 5 non-myositis controls. The dashed line indicates the significance threshold of -log $p$-value $>1.3(p$-value $<0.05)$ for overrepresentation of dysregulated genes in the pathway

Jo1 subgroup analysis highlighted T-helper cell-related pathways which were not significantly dysregulated in the other subgroups (Fig. 2, Additional file 5).

\section{RTqPCR highlighted miR-96-5p and targets}

The two most significantly DE miRNAs $(p<0.001)$, miR-92a-1-5p and miR-96-5p, and two miRNAs DE in our data $(p<0.01)$ which have previously been reported to be DE in IIM, miR-10a-5p and miR-223-3p [12, 13], were chosen for follow up by RTqPCR in a subset of samples with sufficient remaining RNA (6 PM and 5 DM (including 5 anti-Jo-1 positive), 4 IBM, and 4 controls). MiR-96-5p had the highest degree of differential expression, upregulated for PM, DM and anti-Jo1, consistent with the small RNA sequencing data, although the RTqPCR data was only statistically significant for DM, probably due to small sample size (Additional Table 2). MiR-10a-5p was validated as significantly downregulated in IBM. MiR-92a-1-5p and miR-223-3p were not significantly DE in RTqPCR analysis of these samples.
Predicted targets of miR-96-5p which were downregulated in the RNA sequencing data were identified using the IPA microRNA target filter tool (Fig. 3). A subset of the mRNAs identified with high confidence as predicted targets ( $A D K$ and $D A B 1)$ or the most significant dysregulation in the RNA sequencing data (SLC4A1O and CD28) (Fig. 3c) were measured by RTqPCR in samples with remaining RNA (5 PM, 5 DM, (4 anti-Jo1 positive) and 4 controls). $A D K$ and $C D 28$ were significantly downregulated in anti-Jo1 samples, while SLC4A10 was significantly downregulated across the PM, DM and anti-Jo-1 subgroups $(p<0.05$, Table 2$)$. DAB1 was not expressed at a detectable level.

Predicted targets of miR-96-5p which were downregulated in our PM and anti-Jo1 subgroup RNA sequencing data were identified using the IPA microRNA target filter tool and measured by RTqPCR in samples with further remaining RNA (5 PM, 5 DM, (4 anti-Jo1 positive) and 4 controls). Expression fold change was calculated using the 2- $\Delta \Delta \mathrm{Ct}$ method and then converted to $\log _{2}$ 

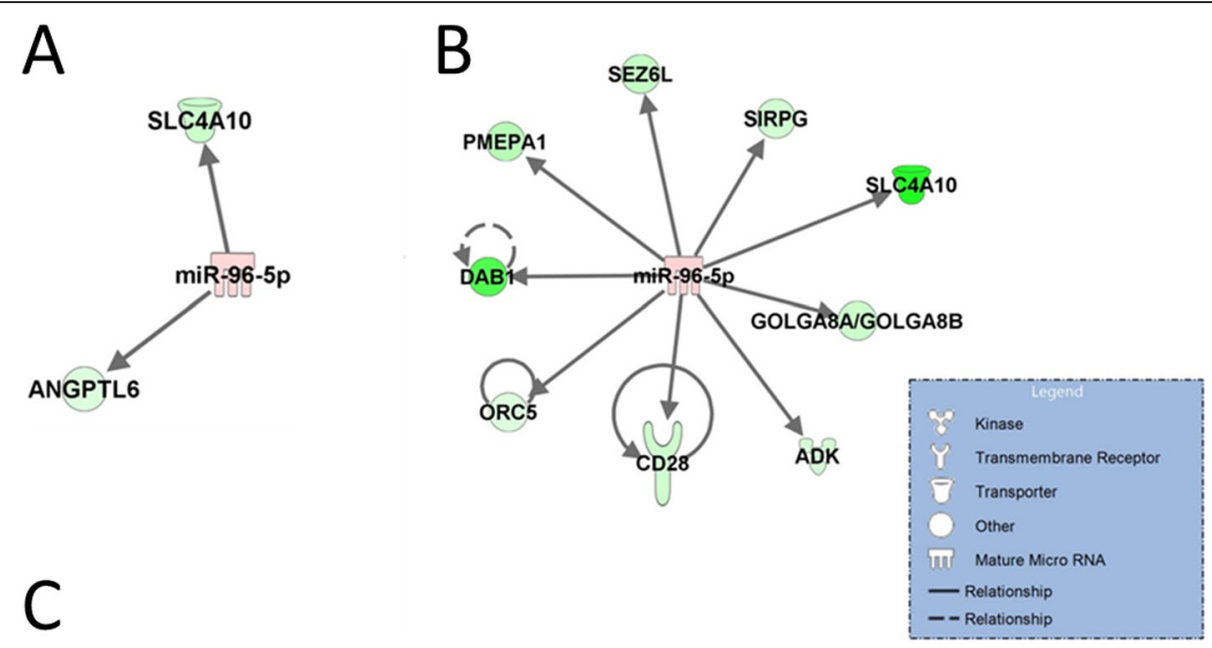

\begin{tabular}{|l|l|l|l|l|l|}
\hline Subgroup & \multicolumn{2}{|l|}{ IPA microRNA target filter } & TargetScan & \multicolumn{2}{l|}{ RNAseq } \\
\cline { 2 - 6 } & Target Gene & Confidence & $\begin{array}{l}\text { Cumulative } \\
\text { weighted context } \\
+ \text { score }\end{array}$ & $\begin{array}{l}\text { Log }_{2} \text { fold } \\
\text { change }\end{array}$ & $\begin{array}{l}\text { False discovery } \\
\text { rate }\end{array}$ \\
\hline \multirow{4}{*}{ PM } & ANGPTL6 & Moderate & -0.261 & -1.95 & 0.0456 \\
\cline { 2 - 6 } & SLC4A10 & Moderate & -0.270 & -5.12 & 0.0002 \\
\hline \multirow{5}{*}{ Anti-J01 } & ADK & High & -0.422 & -1.16 & 0.0337 \\
\cline { 2 - 6 } & CD28 & Moderate & -0.294 & -1.34 & 0.0175 \\
\cline { 2 - 6 } & DAB1 & High & -0.432 & -4.84 & 0.0009 \\
\cline { 2 - 6 } & GOLGA8A & Moderate & -0.289 & -0.94 & 0.3140 \\
\cline { 2 - 6 } & GOLGA8B & Moderate & -0.316 & -1.39 & 0.0463 \\
\cline { 2 - 6 } & ORC5 & Moderate & -0.219 & -0.85 & 0.0438 \\
\cline { 2 - 6 } & PMEPA1 & Moderate & -0.287 & -1.95 & 0.0176 \\
\cline { 2 - 6 } & SE26L & Moderate & -0.383 & -1.64 & 0.0419 \\
\cline { 2 - 6 } & SIRPG & Moderate & -0.213 & -1.23 & 0.0465 \\
\cline { 2 - 6 } & SLC4A10 & Moderate & -0.270 & -8.86 & $8.36 \mathrm{E}-11$ \\
\hline
\end{tabular}

Fig. 3 Networks of predicted targets of miR-96-5p downregulated in idiopathic inflammatory myopathy patients compared to controls QIAGEN's Ingenuity Pathway Analysis (IPA) 'microRNA target filter' predicted mRNA targets of miR-96-5p which are downregulated in RNA sequencing of whole blood from (a) polymyositis (PM) $(n=7)$ and (b) anti-Jo1 positive PM and dermatomyositis (DM) samples $(n=5)$ compared to controls $(n=5)$. No targets were identified in the DM subgroup. Red colouring indicates upregulation, green colouring indicates downregulation and darker shades indicate greater fold change. (c) Summary of IPA microRNA target filter predicted targets. Confidence is 'high' if the TargetScan database cumulative weighted context ++ score (CWCS) is -0.4 or below, indicating a predicted repression of at least $25 \%$ and 'moderate' if the CWCS is -0.2 to -0.4 , indicating a predicted repression of $13-25 \%$

fold change values. $P$-values were calculated using an independent $\mathrm{T}$-test on delta $\mathrm{Ct}$ values from each sample (Experimental Ct-Reference $\mathrm{Ct}$ ) in the subgroups.

\section{Transfection of miR-96-5p mimic resulted in downregulation of $A D K$}

To investigate the impact of miR-96-5p on the expression of predicted mRNA targets downregulated in IIM in a cell culture model relevant to IIM, human skeletal muscle cells were transfected with miR-96-5p or negative control miRNA mimic. After $24 \mathrm{~h}$, RNA was isolated from cells and expression of miR-96-5p targets $A D K$, CD28 and SLC4A1O was measured using RTqPCR.
Expression of $A D K$, but not $C D 28$ or SLC4A10, was significantly downregulated in cells transfected with miR96-5p $(n=5)$ compared to cells transfected with negative control mimic $(n=3)$ suggesting that $A D K$ is suppressed by miR-96-5p in human skeletal muscle (Additional Table 3).

\section{Discussion}

To our knowledge, this is the first study to simultaneously profile both miRNA and mRNA in IIM whole blood using next generation RNA sequencing. Pathway analyses highlighted enrichment of DE genes in innate immune and anti-viral response pathways in PM and 
Table 2 RTqPCR of predicted miR-96-5p mRNA targets identified in RNA sequencing in IIM patients versus controls

\begin{tabular}{|c|c|c|c|c|c|c|}
\hline & \multirow[t]{3}{*}{ Subgroup } & \multicolumn{3}{|c|}{ RTqPCR } & \multicolumn{2}{|l|}{ RNAseq } \\
\hline & & \multicolumn{2}{|c|}{$\log _{2}$ fold change } & \multirow{2}{*}{$\begin{array}{l}\text { T-test } \\
\boldsymbol{P} \text {-value }\end{array}$} & \multirow{2}{*}{$\begin{array}{l}\log _{2} \\
\text { fold } \\
\text { change }\end{array}$} & \multirow[t]{2}{*}{ FDR } \\
\hline & & Mean & \pm & & & \\
\hline \multirow[t]{3}{*}{$\overline{A D K}$} & PM & -0.64 & 0.87 & 0.0690 & -0.75 & 0.3520 \\
\hline & DM & -0.49 & 0.78 & 0.0960 & -0.56 & 0.8960 \\
\hline & Anti-Jo1 & -0.82 & 0.63 & 0.0290 & -1.16 & 0.0337 \\
\hline \multirow[t]{3}{*}{ CD28 } & PM & -1.12 & 1.59 & 0.1240 & -0.91 & 0.2780 \\
\hline & DM & -0.63 & 1.01 & 0.2840 & -0.55 & 1.0000 \\
\hline & Anti-Jo1 & -1.50 & 1.21 & 0.0340 & -1.34 & 0.0175 \\
\hline \multirow[t]{3}{*}{ SLC4A10 } & PM & -4.62 & 1.89 & 0.0010 & -5.12 & 0.0002 \\
\hline & DM & -4.02 & 1.25 & 0.0010 & -2.36 & 1.0000 \\
\hline & Anti-Jo1 & -5.28 & 0.96 & $2.40 \mathrm{E}-05$ & -8.86 & $8.36 \mathrm{E}-11$ \\
\hline
\end{tabular}

PM Polymyositis, DM Dermatomyositis, Anti-Jo1 Subset of PM and DM with anti-Jo1 autoantibodies

DM. Genes in the interferon signalling pathway were upregulated in PM and DM with an increased number of upregulated genes and increased predicted pathway activation in the anti-Jo1 positive subset. This is consistent with previous findings that an interferon score (based on expression of 8 interferon response genes) was higher in whole blood from IIM patients with autoantibodies against RNA-binding proteins, including anti-Jo1, compared to IIM patients with other or no autoantibodies [14]. A larger proportion of the upregulated genes in the anti-Jo1 group were from the type II interferon pathway which is consistent with reports that anti-synthetase syndrome is associated with a prominent type II IFN $\gamma$ signature in muscle biopsies $[15,16]$.

The overall number of significant DE genes was much higher in the anti-Jo1 positive subset than in the clinical groups compared to controls. Anti-Jo1 positivity in IIM is associated with a well-defined set of symptoms, termed anti-synthetase syndrome. Therefore, the higher number of DE genes in the anti-Jo1 positive subset may be due in part to a more homogenous sample allowing for more significant differences to be identified when compared to controls. We do not have data on features such as disease activity, disease manifestations and treatments at time of blood draw which may contribute to the striking transcriptomic profile in this subset. T-helper (Th) cell pathways were significantly enriched for dysregulation in the anti-Jo1 subset but not for any of the clinical groups. Since this analysis was performed on whole blood, these findings may reflect greater differences in cell composition in samples from the antiJo1 positive group compared to other IIM groups and healthy controls. Analysis of differential gene expression in T-cell subsets from different serological groups within IIM may help to elucidate the role of T-helper cells. RNA sequencing in CD4+ and CD8+ T-cells from PM and DM peripheral blood has shown differential gene expression between PM and DM, especially for CD8+ cells, although the contribution of autoantibody positivity to the differential expression was not investigated [17].

In this study, whole blood was chosen as an abundant source of RNA which may reflect systemic changes in circulating immune cells in IIM. Further work is required to assess how these changes affect the main target tissue of IIM, skeletal muscle, and whether changes in circulating microRNA in particular reflect changes in expression in muscle tissue.

MicroRNA profiling identified 4, 4, 7 and 3 DE miRNAs for PM, DM, IBM and the anti-Jo1 positive subset, respectively, with miR-96-5p significantly DE in PM, DM and anti-Jo1 patients. To our knowledge this is the first miRNA profile in IIM whole blood and therefore it is not surprising that we found significantly differentially expressed microRNA that had not been previously identified in IIM in other tissues. Studies have identified serum miR-7 downregulation as a possible biomarker of $\mathrm{PM} / \mathrm{DM}$ which is further reduced in patients with ILD $[18,19]$. This miRNA may not have been identified in our samples due to differential expression between cell types within whole blood. For example, a study in Sjögren's syndrome found that miR-146a-5p was upregulated in T-cells but downregulated in B-cells [20] and a study in systemic sclerosis found that miR-618 was upregulated in plasmacytoid dendritic cells but not significantly DE in monocytes [21]. Therefore, to establish which cell type within whole blood is driving these expression changes and whether they reflect changes in expression in skeletal muscle, expression of the identified DE miRNA and mRNA should be measured in different sample types such as plasma, serum and sorted peripheral blood mononuclear cells.

RTqPCR using samples with remaining RNA for four miRNAs found differential expression consistent with the RNA sequencing results for miR-96-5p and miR10a-5p, although upregulation of miR-96-5p only reached statistical significance for $\mathrm{DM}$, possibly due to small sample sizes. Since miR-96-5p had the largest fold changes in the RTqPCR analyses and has not been identified in IIM before, we investigated the predicted targets of miR-96-5p which were downregulated in the RNA sequencing data. ADK, SLC4A10 and CD28 were validated as downregulated in the anti-Jo1 subgroup by RTqPCR. Transfection of human skeletal muscle cells with a miR96-5p mimic resulted in downregulation of $A D K$ but not SLC4A10 or CD28 expression, suggesting that miR-96$5 \mathrm{p}$ binds and induces degradation of $A D K$ in human 
skeletal muscle. However, binding should be confirmed experimentally and expression analysed in a larger number of whole blood samples and in other tissue types including myositis muscle and sorted blood cells.

miR-96-5p has mainly been studied as an oncogenic miRNA dysregulated in a variety of cancer types including gastric, breast, ovarian and colorectal cancer [2225]. Adenosine Kinase (ADK) is an enzyme that converts adenosine to adenosine monophosphate and adenosine diphosphate. Both upregulation of miR-96-5p and deficiency of $A D K$ have previously been suggested to be associated with mitochondrial dysfunction [26, 27]. Modified reactive oxygen species production as a result of mitochondrial dysfunction may be associated with weakness in IIM [28]. Therefore, if miR-96-5p targeting of ADK is confirmed, this may be an interesting avenue for research in IIM.

The main limitation of this study was the limited sample size due to conservative use of samples from this rare disease. Other limitations include the use of whole blood with variable cellular composition, the higher proportion of males in the control group and the use of samples from patients who have varying degrees of disease severity and were on various treatments for IIM due to the delay to IIM diagnosis, all of which may alter mRNA and miRNA expression.

\section{Conclusions}

Overall, in this study pathway analyses have highlighted enrichment of DE genes in innate immune and anti-viral response pathways in PM and DM and replicated previous findings that interferon signalling is upregulated in PM and DM and more highly upregulated and shifted towards type II interferon signalling in the anti-Jo1 subgroup $[14,15]$. In addition, this study highlighted enrichment of dysregulated genes in the T-helper cell pathways in the anti-Jo1 positive subset and indicated a possible role for miR-96-5p regulation of $A D K$ in pathogenesis of IIM.

\section{Supplementary information}

Supplementary information accompanies this paper at https://doi.org/10. 1186/s41927-020-00125-8.

Additional file 1. Supplementary Methods and Results

Additional file $\mathbf{2}$ Significant RNA sequencing differential expression in polymyositis, dermatomyositis and inclusion body myositis patients versus controls PM Polymyositis, DM Dermatomyositis, IBM Inclusion body myositis, Anti-Jo 1 Subset of PM and DM with anti-Jo1 autoantibodies Data generated by DESeq2 analysis of RNA sequencing data from 7 PM, 7 DM, 5 IBM compared to 5 control whole blood samples. The table columns are gene ids, $\log _{2}$ fold change, standard error for $\log _{2}$ fold change (IfCSE), statistic value for the null hypothesis (stat), $p$-value, false discovery rate (padj), and gene name.

Additional file $\mathbf{3}$ Significant microRNA sequencing differential expression in polymyositis, dermatomyositis and inclusion body myositis patients versus controls PM Polymyositis, DM Dermatomyositis, IBM Inclusion body myositis, Anti-Jol Subset of PM and DM with anti-Jo1 autoantibodies Data generated by DESeq2 analysis of small RNA sequencing data from 7 PM, 7 DM, 5 IBM compared to 5 control whole blood samples. The table columns are mature microRNA ids, $\log _{2}$ fold change, standard error for $\log _{2}$ fold change (IfCSE), statistic value for the null hypothesis (stat), $p$-value, false discovery rate (padj), and mature microRNA name.

Additional file $\mathbf{4}$ GOseq analysis of differentially expressed genes in idiopathic inflammatory myopathy patients versus controls PM Polymyositis, DM Dermatomyositis, IBM Inclusion body myositis, Anti-Jo1 Subset of PM and DM with anti-Jo1 autoantibodies GOseq analysis was performed on significantly differentially expressed genes $(F D R<0.05)$ from RNA sequencing of 7 PM, 7 DM, 5 IBM and 5 control whole blood samples. The anti-Jo1 subgroup consists of 4 PM and 1 DM sample positive for anti-Jo1 autoantibodies. Columns are gene ontology category, $p$ value for over-representation, Benjamini Hochberg adjusted $p$-value for over-representation (FDR $<0.05$ considered significant), $p$-value for underrepresentation, number of differentially expressed genes in that category, total number of genes in the gene ontology category, description of gene ontology category (term) and type of ontology (BP biological process, MF molecular function, CC cellular component).

Additional file $\mathbf{5}$ Canonical Pathways with an enrichment of significantly differentially expressed genes in idiopathic inflammatory myopathy patients PM Polymyositis, DM Dermatomyositis, IBM Inclusion body myositis, Anti-Jo1 Subset of PM and DM with anti-Jo1 autoantibodies QIAGEN's Ingenuity Pathway Analysis (IPA) 'Canonical Pathways' analysis was performed on differential expression results from RNA sequencing of 7 PM and 7 DM (5 anti-Jo1 autoantibody positive), 5 IBM and 5 control whole blood samples. Columns are IPA defined canonical pathway, $-\log (p$-value) for over-representation of dysregulated molecules in the pathway (> 1.3 is considered significant), ratio of dysregulated molecules to total molecules in pathway, activation score ( $z$-score $>2$ is significant predicted activation, $<-2$ is significant predicted inhibition, not determined (ND) when IPA algorithm unable to generate a score) and a list of dysregulated molecules in the pathway.

Additional file 6: Table S1. Assays used in RTqPCR experiments The assay IDs for the TaqMan ${ }^{\oplus}$ Advanced miRNA assays and gene expression assays used to assess reference genes ('Reference selection'), for validation of microRNA ('miRNA validation') and targets ('mRNA validation') found to be dysregulated in RNA sequencing of whole blood from idiopathic inflammatory myopathy patients compared to controls and used to assess expression in human skeletal muscle cells transfected with miRNA mimic compared to cells transfected with negative control miRNA mimic ('Transfection'). Table S2. RTqPCR to validate microRNA identified in RNA sequencing in idiopathic inflammatory myopathy patients versus controls PM Polymyositis, DM Dermatomyositis, IBM Inclusion body myositis, Anti-Jo1 Subset of PM and DM with anti-Jo1 autoantibodies, IIM Idiopathic inflammatory myopathy, DE Differentially expressed RTqPCR was performed on total RNA from 6 PM and 5 DM (including 5 anti-Jo-1), 4 IBM, and 4 control whole blood samples. The RTqPCR results are presented next to the RNA sequencing results for these microRNA for comparison. Expression fold change was calculated using the $2^{-\Delta \Delta \mathrm{Ct}}$ method and then converted to $\log _{2}$ fold change values. $P$-values were calculated using an independent T-test on delta $\mathrm{Ct}$ values from each sample (Experimental Ct-Reference Ct) in the subgroups. Table S3. RTqPCR results for skeletal muscle cells transfected with miR96-5p mimic compared to controls RTqPCR assays for the expression of predicted miR-96-5p mRNA targets were performed on total RNA extracted from human skeletal muscle cells transfected with miR-96-5p miRNA mimic $(n=5)$ and cells transfected with negative control miRNA mimic $(n=3)$. Expression fold change was calculated using the $2^{-\Delta \Delta c t}$ method and then converted to $\log _{2}$ fold change values. P-values were calculated using an independent T-test on delta $\mathrm{Ct}$ values from each sample (Experimental Ct-Reference $\mathrm{Ct}$ ).

\section{Abbreviations}

IIM: Idiopathic inflammatory myopathy; ADK: Adenosine kinase: cDNA: Complementary DNA; DAB1: DAB adaptor protein 1; DE: Differentially 
expressed; DM: Dermatomyositis; FDR: False discovery rate; IBM: Inclusion body myositis; IPA: Ingenuity pathway analysis; miRNA: MicroRNA; MRTB: Myositis research tissue bank; PM: Polymyositis; PRDM4: PR Domain Zinc Finger Protein 4; RTqPCR: Reverse transcription quantitative polymerase chain reaction; SLC4A10: Solute carrier family 4 member 10; UBE2D2: Ubiquitin conjugating enzyme E2 D2

\section{Acknowledgments}

The cDNA library preparation for mRNA and all sequencing was performed at the Genomic Technology Core Facility at the University of Manchester. Initial analysis of the RNA sequencing data was performed by Dr. Ping Wang at the Bioinformatics Core Facility at the University of Manchester. Access to samples in the Myositis Research Tissue Bank was assisted by Hazel Platt and training provided by Fiona Marriage at the University of Manchester. Immortalized human myoblasts were a kind gift from Dr. Vincent Mouly at the Centre for Research in Myology in Paris, France.

\section{Author contributions}

$J \mathrm{~L}, \mathrm{HC}$ and PD designed and supervised the study. JP contributed to the study design, performed the experimental work and analysed and interpreted the data. AL and AT contributed to the transfection study design and experimental work. All authors critically revised drafts of the article and approved the final version.

\section{Funding}

This study was supported in part by Arthritis Research UK (18474). JP was supported by a University of Manchester alumni "Research Impact" PhD studentship and President's Doctoral Scholar Award. HC and JL are supported by a grant from the Medical Research Council (MR/N003322/1). This report includes independent research supported by the National Institute for Health Research. The views expressed in this publication are those of the author(s) and not necessarily those of the NHS, the National Institute for Health Research or the Department of Health. None of the funders were involved in the design of the study, collection, analysis, and interpretation of data or in writing the manuscript.

\section{Availability of data and materials}

The datasets generated and analysed during the current study are available in NCBI's Gene Expression Omnibus (Edgar et al., 2002) and are accessible through GEO Series accession number GSE125977 (http://www.ncbi.nlm.nih. gov/geo/query/acc.cgi?acc=GSE125977).

\section{Ethics approval and consent to participate}

The Salford Royal NHS Foundation Trust myositis research tissue bank (MRTB) study was approved by the NHS North West - Haydock Research Ethics Committee (15/NW/0826) and all participants gave written informed consent.

\section{Consent for publication}

Not applicable.

\section{Competing interests}

The authors declare that they have no competing interests.

\section{Author details}

'Centre for Epidemiology, Division of Population Health, Health Services Research \& Primary Care, Faculty of Biology, Medicine and Health, Manchester Academic Health Science Centre, University of Manchester, Manchester, UK. ${ }^{2}$ Stopford Building, University of Manchester, Oxford Road M13 9PT, Manchester, UK. ${ }^{3}$ Musculoskeletal Science \& Sports Medicine Research Centre, School of Healthcare Science, Manchester Metropolitan University, Manchester, UK. ${ }^{4}$ Manchester Institute of Biotechnology, University of Manchester, Manchester, UK. ${ }^{5}$ Division of Evolution \& Genomic Sciences, University of Manchester, Manchester, UK. ${ }^{6}$ Centre for Musculoskeletal Research, Division of Musculoskeletal \& Dermatological Sciences, Faculty of Biology, Medicine and Health, Manchester Academic Health Science Centre, University of Manchester, Manchester, UK. ${ }^{7}$ National Institute for Health Research Manchester Biomedical Research Centre, Manchester University NHS Foundation Trust, Manchester Academic Health Science Centre, University of Manchester, Manchester, UK. ${ }^{8}$ Department of Rheumatology,
Salford Royal NHS Foundation Trust, Manchester Academic Health Science Centre, Salford, UK.

Received: 16 October 2019 Accepted: 20 March 2020

Published online: 10 June 2020

\section{References}

1. McHugh NJ, Tansley SL. Autoantibodies in myositis. Nat Rev Rheumatol. 2018;14(5):290-302.

2. Saito $Y$, Saito $H$, Liang G, Friedman JM. Epigenetic alterations and MicroRNA Misexpression in cancer and autoimmune diseases: a critical review. Clin Rev Allergy Immunol. 2014;47(2):128-35.

3. Horak M, Novak J, Bienertova-Vasku J. Muscle-specific microRNAs in skeletal muscle development. Dev Biol. 2016;410(1):1-13.

4. Eisenberg I, Eran A, Nishino I, Moggio M, Lamperti C, Amato AA, et al. Distinctive patterns of microRNA expression in primary muscular disorders. Proc Natl Acad Sci U S A. 2007;104(43):17016-21.

5. Parkes JE, Day PJ, Chinoy H, Lamb JA. The role of microRNAs in the idiopathic inflammatory myopathies. Curr Opin Rheumatol. 2015;27(6): 608-15.

6. Bohan A, Peter JB. Polymyositis and dermatomyositis (first of two parts). N Engl J Med. 1975;292(7):344-7.

7. Bohan A, Peter JB. Polymyositis and dermatomyositis (second of two parts). N Engl J Med. 1975;292(8):403-7.

8. Griggs RC, Askanas V, DiMauro S, Engel A, Karpati G, Mendell JR, et al. Inclusion body myositis and myopathies. Ann Neurol. 1995;38(5):705-13.

9. Rose MR, Group EIW. 188th ENMC international workshop: inclusion body myositis, 2-4 December 2011, Naarden, the Netherlands. Neuromuscul Disord. 2013;23(12):1044-55.

10. Lilleker JB, Vencovsky J, Wang G, Wedderburn LR, Diederichsen LP, Schmidt J, et al. The EuroMyositis registry: an international collaborative tool to facilitate myositis research. Ann Rheum Dis. 2018;77(1):30-9.

11. Abd Al Samid M, JS MP, Saini J, TR MK, Fitzpatrick LM, Mamchaoui K, et al. A functional human motor unit platform engineered from human embryonic stem cells and immortalized skeletal myoblasts. Stem Cells Cloning. 2018;11: $85-93$.

12. Xu D, Huang C, Kachaochana A, Morgan GA, Maria F, Soares MB, et al. MicroRNA-10a regulation of Proinflammatory mediators : an important component of untreated juvenile dermatomyositis. J Rheumatol. 2016;43(1): $161-8$.

13. Inoue $\mathrm{K}$, Jinnin M, Yamane $\mathrm{K}$, Makino T, Kajihara I, Makino K, et al. Down-regulation of miR-223 contributes to the formation of Gottron's papules in dermatomyositis via the induction of PKC $\varepsilon$. Eur J Dermatol. 2013;23(2):160-8

14. Ekholm L, Vosslamber S, Tjärnlund A, De Jong TD, Betteridge Z, Mchugh N, et al. Autoantibody specificities and type I interferon pathway activation in idiopathic inflammatory myopathies. Scand J Immunol. 2016;84(2):100-9.

15. Rigolet M, Hou C, Amer YB, Aouizerate J, Periou B, Gherardi RK, et al. Distinct interferon signatures stratify inflammatory and dysimmune myopathies. RMD Open. 2019:5(1):e000811.

16. Pinal-Fernandez I, Casal-Dominguez M, Derfoul A, Pak K, Plotz P, Miller F, et al. Identification of distinctive interferon gene signatures in different types of myositis. Neurology. 2019;93(12):e1193-204.

17. Houtman M, Ekholm L, Hesselberg E, Chemin K, Malmström V, Reed AM, et al. T-cell transcriptomics from peripheral blood highlights differences between polymyositis and dermatomyositis patients. Arthritis Res Ther. 2018;20(1):188.

18. Oshikawa Y, Jinnin M, Makino T, Kajihara I, Makino K, Honda N, et al. Decreased miR-7 expression in the skin and sera of patients with dermatomyositis. Acta Derm Venereol. 2013;93(3):273-6.

19. Yu L, Li J, Chen Y, Jiang J, Fang Q, Jiang J, et al. hsa-miR-7 is a potential biomarker for idiopathic inflammatory myopathies with interstitial lung disease in humans. Ann Clin Lab Sci. 2018:48(6):764-9.

20. Wang-Renault S-F, Boudaoud S, Nocturne G, Roche E, Sigrist N, Daviaud C, et al. Deregulation of microRNA expression in purified T and B lymphocytes from patients with primary Sjögren's syndrome. Ann Rheum Dis. 2018;77: 133-40 Available from: http://ard.bmj.com/lookup/doi/10.1136/ annrheumdis-2017-211417.

21. Rossato M, Affandi AJ, Thordardottir S, Wichers CGK, Cossu M, Broen JC a, et al. Association of MicroRNA-618 expression with altered frequency and activation of Plasmacytoid dendritic cells in patients with systemic sclerosis. 
Arthritis Rheumatol. 2017;69(9):1891-902Available from:. https://doi.org/10, 1002/art.40163.

22. He X, Zou K. MiRNA-96-5p contributed to the proliferation of gastric cancer cells by targeting FOXO3. J Biochem. 2020;167(1):101-8.

23. Gao X, Zhang Y, Zhang Z, Guo S, Chen X, Guo Y. MicroRNA-96-5p represses breast cancer proliferation and invasion through Wnt/B-catenin signaling via targeting CTNND1. Sci Rep. 2020;10(1):44.

24. Liu B, Zhang J, Yang D. miR-96-5p promotes the proliferation and migration of ovarian cancer cells by suppressing Caveolae1. J Ovarian Res. 2019;12(1): 57.

25. Ress A, Stiegelbauer V, Winter E, Schwarzenbacher D, Kiesslich T, Lax S, et al. MiR-96-5p influences cellular growth and is associated with poor survival in colorectal cancer patients. Mol Carcinog. 2015;54(11):1442-50.

26. Jeong HJ, Park SY, Yang WM, Lee W. The induction of miR-96 by mitochondrial dysfunction causes impaired glycogen synthesis through translational repression of IRS-1 in SK-Hep1 cells. Biochem Biophys Res Commun. 2013:434(3):503-8.

27. Boison D, Scheurer L, Zumsteg V, Rülicke T, Litynski P, Fowler B, et al. Neonatal hepatic steatosis by disruption of the adenosine kinase gene. Proc Natl Acad Sci U S A. 2002;99(10):6985-90.

28. Lightfoot AP, Nagaraju K, MCArdle A, Cooper RG. Understanding the origin of non-immune cellmediated weakness in the idiopathic inflammatory myopathies - potential role of ER stress pathways. Curr Opin Rheumatol. 2015;27(6):580-5.

\section{Publisher's Note}

Springer Nature remains neutral with regard to jurisdictional claims in published maps and institutional affiliations.

Ready to submit your research? Choose BMC and benefit from:

- fast, convenient online submission

- thorough peer review by experienced researchers in your field

- rapid publication on acceptance

- support for research data, including large and complex data types

- gold Open Access which fosters wider collaboration and increased citations

- maximum visibility for your research: over $100 \mathrm{M}$ website views per year

At $\mathrm{BMC}$, research is always in progress.

Learn more biomedcentral.com/submissions 